\title{
Xanthogranulomatous cholecystitis presenting as empyema gallbladder in patient of chronic cholecystitis with cholelithiasis
}

\begin{abstract}
Xanthogranulomatous cholecystitis (XGC) is an unusual histopathological variety of chronic cholecystitis, primarily characterized by focal or diffuse inflammation of gallbladder. The chief presenting features include pain, fever, jaundice, signs of cholangitis etc. Intraoperative findings include dense adhesion around the gallbladder (GB) with the surrounding structures, thick gallbladder wall, and firm adhesion of posterior gallbladder wall to the liver. We present a case of XGC as frank empyema gallbladder with large gallstones. The aim of reporting this case i.e. empyema gallbladder, is was to ensure careful complete removal of such GB at the time of operation as in most of the cases surgeons do not find the clear plane of dissection, owing to dense adhesions all over, for complete cholecystectomy and subtotal cholecystectomy remains the last choice. Since XGC is associated with gallbladder cancer $(\mathrm{GBC})$ in $6-12 \%$ of cases and most of the cases undergo partial or subtotal cholecystectomy, therefore, close follow-up of these patients is crucial and must be adopted as standard practice.
\end{abstract}

Keywords: xanthogranulomatous cholecystitis, chronic cholecystitis, subtotal cholecystectomy, empyema gallbladder, cholelithiasis
Volume 4 Issue I - 2018

\section{Pankaj Srivastava, Shalini Srivastava}

Om Surgical Center \& Maternity Home, India

Correspondence: Pankaj Srivastava, Laparoscopic, Thoracic, Thoracoscopic \& VATS Surgeon Om Surgical Center \& Maternity Home, SA I7/3, P-4, Sri Krishna Nagar, Paharia, Ghazipur Road,Varanasi, UP, India, PIN-22 I007, Tel +91-5422586I9I, +9194I52268I7, Email drpankajbns@gmail.com

Received: January 29, 2018 | Published: February 12, 2018
Abbreviations: XGC, xanthogranulomatous cholecystitis; GBC, gallbladder cancer; RHC, right hypochondrium; GB, gallbladder

\section{Introduction}

Xanthogranulomatous cholecystitis (XGC), a rare form of chronic cholecystitis which fairly mimics gallbladder cancer, was first discovered and reported by McCoy et al. ${ }^{1}$ Although it is considered as benign disease but often reported its close association with gallbladder cancer (GBC). ${ }^{2,3}$ Clinical signs and symptoms are similar to acute and chronic cholecystitis, thus preoperative diagnosis is a big challenge to surgeons and radiologists as well. Cholelithiasis and choledocholithiasis are frequently associated with XGC. Important differential diagnosis is with (GBC) as most of the presenting features are overlapping and mode of treatment varies. Cholecystectomy is the gold standard treatment as in any gallstone disease but due to dense and firm adhesions, only partial or subtotal cholecystectomy remains the choice in most of the cases. Dissection should not proceed by force and the excision range should not be blindly extended in order to avoid injuries to the extrahepatic bile duct and neighboring organs. Special attention should be paid to cases where internal fistula or Mirizzi syndrome is found and biliary injuries should be avoided. ${ }^{4}$

\section{Case presentation}

A 50-years-old non-smoker, non-alcoholic, non-vegetarian male presented with sudden onset of severe pain in right side of upper abdomen for one day when he was in flight for which he took some antacid and got relief. He had a history of recurrent episodes of similar upper abdomen pain for the last two years. The pain was not associated with vomiting and fever. There was no history of jaundice in past two years. He was carpenter by profession working in Gulf. There was no history of any acute or chronic illness and of any surgery.
On examination, abdomen was tender and distended with guarding in right hypochondrium (RHC). Soft to firm globular swelling was palpable in RHC which was tender. No other obvious findings were noticed. His pulse rate was $87 / \mathrm{min}$, blood pressure in lying posture was $132 / 82 \mathrm{mmHg}$, respiratory rate was $20 / \mathrm{min}$, oral temperature was $98.60 \mathrm{~F}, \mathrm{SPO} 2$ was $99 \%$ in room air. Other systemic examinations were normal. Ultrasonography of abdomen revealed features suggestive of chronic cholecystitis with cholelithiasis with thickened gallbladder $(\sim 8 \mathrm{~mm})$ (Figure 1$)$. Common bile duct was of normal caliber with no echogenic shadow within it. Other abdominal organs were within normal range. Chest X-ray and ECG were also normal. The leukocytosis $(13900 /$ cumm) with neutrophilia $(86 \%)$ was present. Other biochemistry and serology investigations were within normal range. On the basis of history and clinical findings, patient was diagnosed as a case of acute or chronic cholecystitis with cholelithiasis.

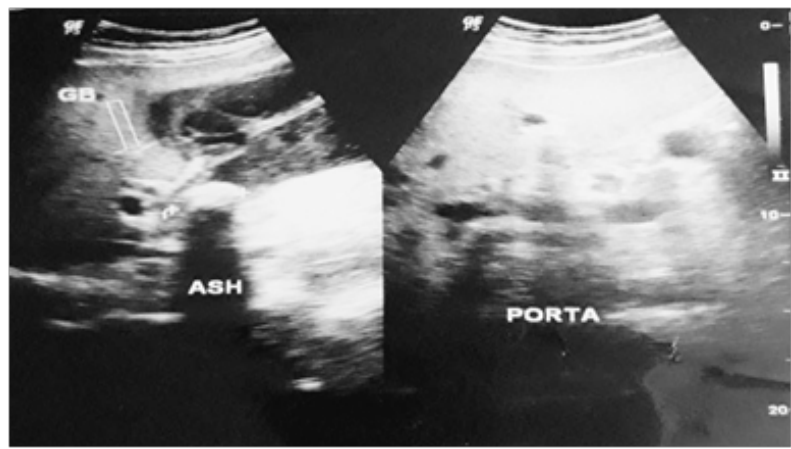

Figure I Ultrasonography revealed thickened gallbladder with multiple large gallstones with normal porta.

Title USG of xanthogranulomatous cholecystitis patient. 
Under general anesthesia, laparoscopic cholecystectomy was planned. Patient was kept supine and standard four ports were introduced. Initial examination of abdomen revealed dense adhesions around the gallbladder. Adhesiolysis was tried but failed as bowel was firmly adhered to the GB and GB wall was very fragile, therefore, conversion was decided. Patient was then opened through right Kocher's incision. After entering the abdomen, meticulous adhesiolysis was again performed, during which GB got opened and thick blood mixed pus came out. All the pus was aspirated and stones were removed thoroughly. GB lumen was irrigated with normal saline several times to takeout any residual stone. After that, all the bowel adhesions were removed by blunt and sharp dissection. Gallbladder was removed from the liver bed up to the neck and then subtotal cholecystectomy was done as anatomy of Calot's triangle was obscure. Dissection area was thoroughly washed with Luke warm normal saline. $18 \mathrm{Fr}$ abdominal drains were put near the GB fossa and abdomen was then closed in layers. Patient was extubated on table. GB specimen was sent for histopathology examination. No intraoperative complication noticed.

Postoperative period was uneventful. Abdominal drain was out on day 4 and stitches were removed on day 8 . Patient was discharged on day 9. Follow-up period after 15 days and two months were uneventful. Patient was advised for the regular follow-up.

Gross pathologic examination of GB revealed $5.5 \mathrm{~cm}$ long, $6.0 \mathrm{~cm}$ maximum diameter tissue. No lymph node. The serosa of the GB was tan-brown, smooth and shiny. The hepatic surface was also tan-brown. The GB wall ranged from $0.8-1.0 \mathrm{~cm}$ in thickness and was grossly unremarkable. The mucosa was green and velvety, without polyps or other lesions. Microscopic examination revealed GB mucosa with focal ulceration. Lamina propria showed acute and chronic inflammatory cell infiltrate comprised of neutrophils, lymphocytes, and plasma cells. There were sheets of foamy histiocytes and few hemosiderin-laden macrophages but no evidence of granuloma or malignancy (Figure 2).

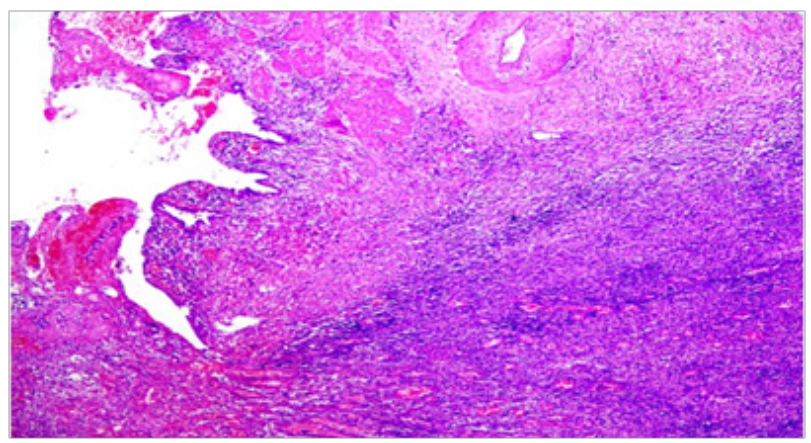

Figure 2 Microscopic examination revealed GB mucosa with focal ulceration. Lamina propria showed acute and chronic inflammatory cell infiltrate comprised of neutrophils, lymphocytes, and plasma cells. There were sheets of foamy histiocytes and few hemosiderin-laden macrophages but no evidence of granuloma or malignancy.

Title Low power view of xanthogranulomatous gallbladder (H\&E stain).

\section{Discussion}

Xanthogranulomatous cholecystitis (XGC) is entirely histopathogic diagnosis. Preoperative diagnosis poses challenge to surgeons and radiologists as there is no pathognomonic feature of XGC. The most pertinent differential diagnosis of $\mathrm{XGC}$ is $\mathrm{GBC}$ as $\mathrm{XGC}$ is often mimicking the features of GB cancer and is also associated with that too. ${ }^{2,3}$ Since its first report by McCoy et al. ${ }^{1}$ lot of publications have enriched the medical literature but no conclusive message regarding its preoperative diagnosis, management could be drawn with success. Its pathogenesis consist some relation between bile flow obstruction with infection and subsequent inflammation associated with leakage of bile to tissues where histiocytes accumulate and phagocytose the bile pigment, hemosiderin and cholesterol, resulting in the formation of xanthoma cells. ${ }^{5}$ No clinical feature is specific to XGC; clinical presentation is similar to those of acute or chronic cholecystitis. No specific radiological feature is associated with XGC but few are strongly indicative as uniform diffuse thickening of the GB wall, adhesions to the adjacent structures and organs, percholecystic fluid, and intramural nodules and bands. Gall stones are strongly associated with XGC but are not present in every patient, indicating a role for additional etiological factors. Hale et al. ${ }^{6}$ suggested that there may be a geographical influence upon the incidence of XGC with rates being approximately three to four times greater in India than in other geographical regions owing to increased incidence of gall stones in India. Gall stones are believed to be causative of XGC owing to causing obstruction of the cystic duct, biliary stasis and gall bladder mucosa ulceration, all of which facilitate local inflammation and extravasation of bile into the gallbladder wall. ${ }^{6}$

Priya et al. ${ }^{7}$ revealed a higher incidence of loss of heterozygosity (LOH) of the fragile histadine triad tumor suppressor gene in XGC compared with chronic cholecystitis implicates a genetic basis for XGC and a link with GBC (in which LOH was not significantly greater than in XGC). Although cholecystectomy remains the treatment of choice but complete cholecystectomy is difficult to achieve in majority of cases. Subtotal or partial cholecystectomy usually suffices the purpose. Postoperative complications like bile leakage, biliary peritonitis, bleeding, abscess formation, wound infections, cholangitis, and stenosis are more associated with XGC patients. Above all, meticulous operative technique, sound knowledge of biliary anatomy and proper postoperative care suffice the ultimate patient care of XGC patients.

\section{Conclusion}

$\mathrm{XGC}$ is a rare pathologic entity, usually presenting as chronic cholecystitis with or without gallstones. Empyema gallbladder is not an uncommon operative finding. Most of the time, such gallbladders are very difficult to take out In toto because of dense and firm adhesions with the important surrounding structures. But having association with the XGC, one should try to be very meticulous during surgery and every effort must be made to excise most of the GB if not complete possible. It is also advisable to cauterize the mucosa when it cannot be completely excised. Since pathogenesis is still obscure further efforts are required to get more information. Histopathologic markers are required to make confirm preoperative diagnosis. All these efforts should be made to differentiate XGC from the GBC so that over-treatment with unnecessary major surgery or undertreatment for missed GBC can be avoided. Microscopic examination revealed GB mucosa with focal ulceration. Lamina propria showed acute and chronic inflammatory cell infiltrate comprised of neutrophils, lymphocytes, and plasma cells. There were sheets of foamy histiocytes and few hemosiderin-laden macrophages but no evidence of granuloma or malignancy.

\section{Acknowledgements}

\section{None.}




\section{Conflict of interest}

Author declares that there is no conflict of interest.

\section{References}

1. McCoy JJ, Vila R, Petrossian G, et al. Xanthogranulomatous cholecystitis Report of two cases. J S C Med Assoc. 1976;72(3):78-79.

2. Rao RV, Kumar A, Sikora SS, et al. Xanthogranulomatous cholecystitis: differentiation from associated gall bladder carcinoma. Trop Gastroenterol. 2005;26(1):31-33.

3. Ghosh M, Sakhuja P, Agarwal AK. Xanthogranulomatous cholecystitis: a premalignant condition? Hepatobiliary Pancreat Dis Int. 2011;10(2):179-184.
4. Alhomoud H, Abdelmohsen M. Xanthogranulomatous Cholecystitis. World J Lap Surg. 2017;10(2):77-79.

5. Mulchandani M, Nagori LF. Xanthogranulomatous cholecystitis. Bombay Hospital Journal. 2000;42(4):31-32.

6. Hale MD, Roberts KJ, Hodson J, et al. Xanthogranulomatous cholecystitis: a European and global perspective. HPB. 2014;16(5):448-458.

7. Priya TP, Kapoor VK, Krishnani N, et al. Fragile histidine triad (FHIT) gene and its association with $\mathrm{p} 53$ protein expression in the progression of gall bladder cancer. Cancer Invest. 2009;27(7):764-773. 